\title{
Residual properties of a two-level system
}

\author{
J. J. Brey and A. Prados \\ Física Teórica, Universidad de Sevilla, Apartado 1065, 41080 Sevilla, Spain \\ (Received 10 September 1990; revised manuscript received 16 November 1990)
}

\begin{abstract}
The residual properties, after cooling from a high temperature to $T=0 \mathrm{~K}$, of a system of identical and independent two-level systems are analyzed. A general criterion to know whether a given cooling law will lead to a nonvanishing residual population is derived. After defining in a precise way the condition of slow cooling, asymptotic expressions for the residual population and entropy are obtained for a family of cooling laws. It is shown that the expressions for the residual properties, and their own existence, depend quite strongly on the cooling procedure.
\end{abstract}

\section{INTRODUCTION}

One of the most used and faithful methods for modeling the static properties of glasses at low temperatures is by means of an ensemble of two-level systems. ${ }^{1}$ In the last few years, the same kind of approximation is being used as a model for the high-temperature dynamical properties of frustrated systems. Huse and Fisher ${ }^{2}$ have proposed that the residual energy, after slow cooling from high temperatures to $T=0 \mathrm{~K}$, of various disordered systems can be explained by considering an ensemble of independent two-level systems (TLS), with a given distribution of excitation energies and barriers. A crucial point in their arguments is that the residual energy of a TLS, in the limit of a large cooling time, is roughly independent of the cooling procedure.

Langer et $a l .{ }^{3-5}$ have studied the residual energy and entropy of an ensemble of identical TLS cooled asymptotically slow. They consider two specific cooling laws, and the results they find for the residual energy are equivalent if the cooling rate parameter is appropriately defined for each case. Neverthetless, both cooling schedules are quite different from a physical point of view: one of them requires an infinite time to reach the temperature $T=0$ $\mathrm{K}$, with independence of the value of the cooling rate parameter, while the other one corresponds to a linear decrease of the temperature.

In this paper we consider again an ensemble of independent and identical TLS that are cooled from high temperature to $T=0 \mathrm{~K}$. Our aim is to try to clarify the following points: (a) Does the existence of a nonvanishing residual population depend on the cooling procedure?; (b) If it does, is there a general criterion to determine whether a given cooling law will lead to the existence of a residual energy?; (c) What does slow cooling mean in general?, and (d) How does the value of the residual energy depend on the cooling law and the cooling rate in the limit of slow cooling?.

Another important residual property is the entropy. Once the distribution at $T=0 \mathrm{~K}$ is known, the statistical entropy can be easily computed from its own definition. Nevertheless, as Langer and Sethna ${ }^{3}$ have pointed out, what is measured in actual experiments is a calorimetric entropy, obtained from the heat exchange of the system.
For nonequilibrium systems both definitions are not equivalent. Therefore, it must be distinguished between residual statistical entropy and residual calorimetric entropy. By means of thermodynamical arguments, Langer and Sethna ${ }^{3}$ have shown that the residual calorimetric entropy provides a lower bound for the statistical one. The same result has been derived in Refs. 5 and 6 starting from a general master-equation description of the cooling process. Here, we will compute both residual entropies in the limit of slow cooling for a wide class of cooling laws, and the relation between them will be explicitly established.

From a formal point of view, the calculations we present differ from those in Refs. 2 and 4. There, singular perturbation theory was used to obtain approximate solutions, whereas here we will start from exact solutions, where the interesting limits will be considered.

The plan of the paper is as follows. In Sec. II the model and the notation are introduced. Also, the general solution of the master equation describing the model is given. This expression is used in Sec. III to derive a necessary and sufficient condition for the existence of residual population or energy after continuous cooling to $T=0 \mathrm{~K}$. For those cases that do not lead to residual population we will analyze the way in which the population goes to zero as the temperature decreases. Also, a precise definition of slow cooling will be given and its relation with the cooling rate parameter will be discussed.

The above results are applied in Sec. IV to a oneparameter family of cooling laws. It is shown that there is a critical value of the parameter separating cooling processes with and without residual population. All the processes with a finite time of cooling to $T=0 \mathrm{~K}$ lead to the presence of residual population, but it also appears for some of the processes corresponding to an infinite cooling time. The particular case of a linear variation of the temperature does not belong to this family, and it is separately discussed in Sec. V. Explicit expressions for the residual populations in the asymptotic limit of slow cooling are derived in both sections.

Section VI is devoted to the calculation of the residual entropy for the same family of cooling laws as in Sec. IV. Finally, some general conclusions and discussions are presented in Sec. VII. 


\section{DYNAMICS OF THE TWO-LEVEL SYSTEM}

Our model is a simple two-level system (TLS). The difference in energy between the two possible energy states is $\varepsilon$, and the activation barrier separating them, measured from the lower state, is $\varepsilon+V$. The probability $p(t)$ of finding the system in the upper state is assumed to obey the master equation

$$
\begin{gathered}
\frac{d p(t)}{d t}=\alpha\{\exp [-\beta(t)(\varepsilon+V)][1-p(t)] \\
-\exp [-\beta(t) V] p(t)]\},
\end{gathered}
$$

where $\beta(t)=\left[k_{B} T(t)\right]^{-1}, k_{B}$ being the Boltzmann constant and $T(t)$ the time-dependent temperature. The constant $\alpha$ measures the characteristic frequency of jumping, and we will use it to define an adimensional time variable, i.e., we formally take $\alpha=1$.

Introducing the transition probability

$$
\gamma(t)=[-\beta(t) V],
$$

and the asymmetry parameter

$$
\mu=\epsilon / V \text {, }
$$

we can rewrite Eq. (2.1) in the more compact form

$$
\frac{d p(t)}{d t}=\gamma(t)\left\{\gamma(t)^{\mu}-\left[1+\gamma(t)^{\mu}\right] p(t)\right\}
$$

While $\gamma(t)$ is bounded between 0 and $1, \mu$ can take any positive value. If the temperature is a monotonous function of time, the structure of Eq. (2.4) suggests the use of $\gamma$ as independent variable. Then, assuming that the law of variation of the temperature is given by

$$
\frac{d \gamma(t)}{d t}=-r f[\gamma(t)]
$$

with $r>0$, Eq. (2.4) becomes

$$
-r f(\gamma) \frac{d p(\gamma)}{d \gamma}=\gamma\left[\gamma^{\mu}-\left(1+\gamma^{\mu}\right) p(\gamma)\right] .
$$

The parameter $r$ defines the time scale on which $\gamma$ changes. For $r \ll<1$ the transition probability changes very slowly in time. The sign of $f(\gamma)$, that is independent of the value of $\gamma$, defines the nature of the process. If $f>0$ the system is being cooled, while for $f<0$ the system is being heated. The only additional requirement for $f$ is to be continuous or, at least, to have a finite number of discontinuities.

The several cooling laws that have been considered in the bibliography can, of course, be cast in the form (2.5). For instance, the linear law

$$
T(t)=T_{0}-a t,
$$

used by Huse and Fischer, ${ }^{2}$ corresponds to the choices

$$
f(\gamma)=\gamma(\ln \gamma)^{2},
$$

and

$$
r=k_{B} a / V .
$$

The same authors also consider

$$
T(t)=\frac{V}{k_{B}}\left[\ln \frac{t}{1+\mu}\right]^{-1},
$$

that is equivalent to

$$
f(\gamma)=\gamma^{2},
$$

and

$$
r=\frac{1}{1+\mu}
$$

Finally, Langer, Dorsey, and Sethna ${ }^{4}$ have studied the case

$$
T(t)=\frac{T_{0}}{1+R t},
$$

i.e.,

$$
f(\gamma)=\gamma
$$

and

$$
r=\frac{V R}{k_{B} T_{0}} .
$$

Equation (2.6) is a linear first-order differential equation that can be exactly solved for a given initial condition $p\left(\gamma_{0}\right)=p_{0}$, where $\gamma_{0}$ is the value of $\gamma$ corresponding to the initial temperature, i.e., $\gamma_{0}=\exp \left(-V / k_{B} T_{0}\right)$. Of course, $0 \leq p_{0} \leq 1$. The solution is

$$
\begin{aligned}
p(\gamma)= & {\left[p_{0}+\frac{1}{r} \int_{\gamma}^{\gamma_{0}} d y \frac{y^{1+\mu}}{f(y)} \exp \left(\frac{1}{r} \int_{y}^{\gamma_{0}} d z \frac{z+z^{1+\mu}}{f(z)}\right)\right] } \\
& \times \exp \left[-\frac{1}{r} \int_{\gamma}^{\gamma_{0}} d y \frac{y+y^{1+\mu}}{f(y)}\right] .
\end{aligned}
$$

The above expression holds for arbitrary $f(\gamma), p_{0}, \gamma_{0}$, and $\mu$. Once they are specified, the integrals can be evaluated, resorting, if necessary, to numerical techniques. The residual population at $T=0 \mathrm{~K}$ is obtained particularizing Eq. (2.16) for $\gamma=0$.

It is possible to write Eq. (2.16) in a more transparent form. Integration by parts yields

$$
\begin{aligned}
p(\gamma)= & \frac{\gamma^{\mu}}{1+\gamma^{\mu}}+\left[p_{0}-\frac{\gamma_{0}^{\mu}}{1+\gamma_{0}^{\mu}}\right] \\
& \times \exp \left[-\frac{1}{r} \int_{\gamma}^{\gamma_{0}} d y \frac{y+y^{1+\mu}}{f(y)}\right] \\
& +\mu \int_{\gamma}^{\gamma_{0}} d y \frac{y^{\mu-1}}{\left(1+y^{\mu}\right)^{2}} \\
& \times \exp \left[-\frac{1}{r} \int_{\gamma}^{y} d z \frac{z+z^{1+\mu}}{f(z)}\right] .
\end{aligned}
$$

The first term on the right-hand side is the equilibrium value of $p(\gamma)$ for constant temperature. The second one corresponds to the relaxation from a nonequilibrium state towards the equilibrium curve. It identically vanishes if the system was initially at equilibrium. The last term is associated with the (possible) departure from the equilibrium curve. 
In the following section we will derive several asymptotic properties of the TLS, starting from Eq. (2.17).

\section{A CRITERION FOR THE EXISTENCE OF RESIDUAL POPULATION AT $T=0 \mathrm{~K}$}

Let us consider that the TLS is uniformly cooled to $T=0 \mathrm{~K}$ from a given initial temperature $T_{0}$. The time $t_{0}$ required to realize the process will depend on the cooling law, and it can be finite or infinite. For instance, if $f(\gamma)=\gamma^{k}$ it is easily seen that $t_{0}=\infty$ for $k>1$, and $t_{0}=\gamma_{0}^{1-k}[r(1-k)]^{-1}$ for $k<1$. In the case $k=1, \gamma$ decays exponentially in time.

The residual population (RP) for $\mu \neq 0$ will be given, according to Eq. (2.17), by

$$
\begin{aligned}
p(0)= & {\left[p_{0}-\frac{\gamma_{0}^{\mu}}{1+\gamma_{0}^{\mu}}\right] \exp \left[-\frac{1}{r} \int_{0}^{\gamma_{0}} d y \frac{y+y^{1+\mu}}{f(y)}\right] } \\
& +\mu \int_{0}^{\gamma_{0}} d y \frac{y^{\mu-1}}{\left(1+y^{\mu}\right)^{2}} \exp \left[-\frac{1}{r} \int_{0}^{y} d z \frac{z+z^{1+\mu}}{f(z)}\right] .
\end{aligned}
$$

We will characterize a slow cooling process as that leading to a RP that is independent from the initial value $p_{0}$. Equation (3.1) shows that this requires

$$
\frac{1}{r} \int_{0}^{\gamma_{0}} d y \frac{y+y^{1+\mu}}{f(y)} \gg 1
$$

or, equivalently,

$$
\delta^{-1} \equiv \frac{1}{r} \int_{0}^{\gamma_{0}} d y \frac{y}{f(y)} \gg 1
$$

In fact, $\delta$ is the small parameter we will use in our asymptotic analysis. But before that, let us study under which conditions will be $p(0)$, given by Eq. (3.1), different from zero, independently from the value of $p_{0}$. The relevant quantity is

$$
\zeta(x)=\int_{0}^{x} d y \frac{y+y^{1+\mu}}{f(y)},
$$

where $x$ can take any value between $0^{+}$and $\gamma_{0}$. We notice that since we have assumed that the system is cooled down to $T=0 \mathrm{~K}, f(y)$ can not vanish in the interval $0<y \leq \gamma_{0}$. If it would vanish for $\gamma \equiv \gamma_{1}$, it follows from Eq. (2.5) that the temperature would tend asymptotically in time to the value $T_{1}=-V /\left(k_{B} \ln \gamma_{1}\right)$. Therefore, the character convergent or divergent of $\zeta(x)$ does not depend on $x$ in the range of values considered.

If $\zeta(x)$ is finite, it is clear from Eq. (3.1) that the RP is, in general, different from zero. This holds with independence of the values of $p_{0}$ and $\delta$. On the other hand, when $\xi(x)$ diverges, the first term on the right-hand side of Eq. (3.1) vanishes, and to analyze the second one it is convenient to consider it as given by the limiting process

$$
p(0)=\lim _{\gamma \rightarrow 0} p(\gamma),
$$

where

$$
p(\gamma)=\frac{\mu \int_{\gamma}^{\gamma_{0}} d y \frac{y^{\mu-1}}{\left(1+y^{\mu}\right)^{2}} \exp \left[\frac{1}{r} \int_{y}^{\gamma_{0}} d z \frac{z+z^{1+\mu}}{f(z)}\right]}{\exp \left[\frac{1}{r} \int_{\gamma}^{\gamma_{0}} d z \frac{z+z^{1+\mu}}{f(z)}\right]}
$$

Taking into account that $\gamma_{0} \leq 1$ it is easily seen that for $0<x \leq \gamma_{0}$

$$
\eta(x) \leq \zeta(x) \leq 2 \eta(x),
$$

where

$$
\eta(x)=\int_{0}^{x} d y \frac{y}{f(y)},
$$

and, therefore, the divergence of $\xi(x)$ is equivalent to the divergence of $\eta(x)$, and the condition for this to happen is

$$
\lim _{y \rightarrow 0} f(y) y^{-2+\varepsilon}=0
$$

for arbitrary $\varepsilon>0$. Two cases must be distinguished:

$$
\begin{aligned}
& \text { (I) } \lim _{y \rightarrow 0} f(y) y^{-2}=0, \\
& \text { (II) } \lim _{y \rightarrow 0} f(y) y^{-2}=C
\end{aligned}
$$

with $C$ being a constant. In both cases the denominator in Eq. (3.6) diverges. The numerator also diverges in case (I), but in case (II) it can converge or diverge depending on the values of $r$ and $\mu$. When the numerator does not diverge it is clear that $p(0)=0$. If the numerator diverges we can apply the L'Hospital rule, obtaining the same result.

Therefore, for divergent $\eta(x)$, or equivalently when Eq. (3.9) is verified, there is no $\operatorname{RP}$ (for $\mu=0$, it is $p(0)=1 / 2$ ). In this way we have arrived to a well-defined criterion for the existence of residual population, given by the condition

$$
\lim _{y \rightarrow 0} f(y) y^{-2}=\infty,
$$

that does not involve the parameter $r$.

Thus, for instance, it follows that there will be RP in the cases of the cooling laws given by Eqs. (2.7) and (2.13), but not if the TLS is cooled according to Eq. (2.10).

It is easy to understand why it is the shape of $f(\gamma)$ that determines whether there is a nonvanishing RP, whereas the cooling rate parameter $r$ does not play any role in it. When the system is cooled down to $T=0 \mathrm{~K}, \gamma(t)$ goes to zero with independence of the value of $r$. Therefore, the relevant point is not the value of $r$, but whether the time variation of $\gamma$ is fast enough to prevent the system of following the equilibrium curve. In fact, it is easily seen that $\eta(x)$ can be used to define the relevant time scale. Of course, when there is RP, its specific value will depend on the value of $r$.

In the next section we will investigate the behavior of the RP when Eq. (3.12) is verified. In the remainder of this section we are going to study more deeply the case $\eta(x)=\infty$. In particular, we want to determine the way in 
which $p(\gamma)$ vanishes when $\gamma$ approaches zero. The second term on the right-hand side of Eq. (2.17) can be neglected because it decays exponentially, while the first one shows an algebraic behavior. Besides, the former identically vanishes when the system was initially at equilibrium, as it is usually assumed.

To analyze the behavior of $p(\gamma)$ we consider its relative deviation from the equilibrium curve, i.e., we study the asymptotic behavior for $\gamma \rightarrow 0$ of the ratio $B(\gamma)$ between the third and the first terms in Eq. (2.17). A simple calculation shows that

$$
B(\gamma) \sim \frac{B_{1}(\gamma)}{B_{2}(\gamma)}
$$

where

$$
B_{1}(\gamma)=\mu \int_{\gamma}^{\gamma_{0}} d y \frac{y^{\mu-1}}{\left(1+y^{\mu}\right)^{2}} \exp \left[\frac{1}{r} \int_{y}^{\gamma_{0}} d z \frac{z+z^{1+\mu}}{f(z)}\right],
$$

and

$$
B_{2}(\gamma)=\gamma^{\mu} \exp \left[\frac{1}{r} \int_{\gamma}^{\gamma_{0}} d z \frac{z+z^{1+\mu}}{f(z)}\right) .
$$

The two cases given by Eqs. (3.10) and (3.11) must be considered separately. In case $(\mathrm{I}), B(\gamma)$ has the indeterminate form $\infty / \infty$ and application of L'Hospital rule leads to

$$
\lim _{\gamma \rightarrow 0} B(\gamma)=0
$$

and, therefore, the behavior of the population of the upper level for $\gamma$ small enough is given by

$$
p(\gamma) \sim \frac{\gamma^{\mu}}{1+\gamma^{\mu}},
$$

i.e., it tends to zero along the equilibrium curve. In case (II), we have to distinguish between two possibilities. For $\mu<1 / C r$, we arrive again to a form $\infty / \infty$ whose limit is

$$
\lim _{\gamma \rightarrow 0} B(\gamma)=\frac{C r \mu}{1-C r \mu},
$$

and thus

$$
p(\gamma) \sim \frac{1}{1-C r \mu} \frac{\gamma^{\mu}}{1+\gamma^{\mu}} .
$$

For $\mu \geq 1 / C r, \lim _{\gamma \rightarrow 0} B_{2}(\gamma)=0$ and one gets

$$
\lim _{\gamma \rightarrow 0} B(\gamma)=\infty,
$$

and the leading behavior is given by the last term on the right-hand side of Eq. (3.17). In the particular case $f(\gamma)=C \gamma^{2}$ it is found

$$
\begin{aligned}
p(\gamma) \sim & \mu \gamma^{1 / C r}\left[\exp \frac{\gamma^{\mu}}{C r \mu}\right] \\
& \times \int_{\gamma}^{\gamma_{0}} d y \frac{y^{\mu-1-1 / C r}}{\left(1+y^{\mu}\right)^{2}} \exp \left[-\frac{y^{\mu}}{C r \mu}\right) .
\end{aligned}
$$

In conclusion, the population of the upper level goes to zero along the equilibrium curve, except when $f(\gamma) \sim \gamma^{2}$ for $\gamma \rightarrow 0$. In this latter case, it obeys Eq. (3.19) for $\mu<1 / C r$, while for $\mu \geq 1 / C r$ the decay law depends on the initial condition, and also on the concrete form of $f(\gamma)$.

\section{RESIDUAL POPULATION FOR COOLING LAWS OF THE FORM $f(\gamma)=\gamma^{k}$}

The general arguments presented in the previous section will be applied here to cooling laws given by a power of $\gamma$, i.e.,

$$
f(\gamma)=\gamma^{k}
$$

with $k \in \mathbb{R}$. In this case Eq. (2.6) can be easily integrated yielding

$$
\gamma=\left[\gamma_{0}^{1-k}+(k-1) r t\right]^{1 /(1-k)}
$$

for $k \neq 1$, and

$$
\gamma=\gamma_{0} e^{-r t}
$$

for $k=1$.

It follows from the above equations that for $k \geq 1$ it takes an infinite time to cool the system from a finite temperature $T_{0}=-V\left(k_{B} \ln \gamma_{0}\right)^{-1}$ down to $T=0 \mathrm{~K}$. For $k<1$ the cooling time is finite and it is given by

$$
\tau=\frac{\gamma_{0}^{1-k}}{r(1-k)} \text {. }
$$

According to the criterion obtained previously, Eq. (3.12), there will be a nonvanishing $\mathbf{R P}$ for $k<2$. Besides, for $k>2$ the RP tends to zero on the equilibrium curve for small values of $\gamma$. This is clearly illustrated in Fig. 1, where the evolution of the upper level population is shown, for different values of $k$, and fixed values of $r$ and $\mu$. The curves have been obtained by numerical evaluation of the exact expression, as given by Eq. (2.17). The continuous line is the equilibrium curve.

For $k=2$ the behavior is given by Eq. (3.19) or by Eq. (3.21), depending on whether $r<\mu^{-1}$ or $r \geq \mu^{-1}$. In particular, for the cooling law defined by Eq. (2.10) we have

$$
p(\gamma) \sim(1+\mu) \frac{\gamma^{\mu}}{1+\gamma^{\mu}} \sim(1+\mu) \gamma^{\mu} .
$$

Using Eq. (4.2) it is seen that this expression is equivalent to the asymptotic behavior

$$
p(t) \sim(1+\mu)^{1+\mu_{t}-\mu}
$$

for $t \rightarrow \infty$. Equation (4.6) was derived by Huse and Fisher. ${ }^{2}$ It is important to realize that $p(t)$ is not the RP of the TLS when it is cooled to $T=0 \mathrm{~K}$ in a finite time $t$ following Eq. (2.10). As we have already discussed, and it is clearly shown by Eq. (4.5), the temperature $T=0 \mathrm{~K}$ is reached for $t \rightarrow \infty$, and in this limit $p(t)$ vanishes. We cannot properly speak of RP for finite $t$ in this case.

Let us proceed now to compute the RP in the case $k<2$. Particularizing Eq. (3.1) for the law given by Eq. (4.1) it is found 


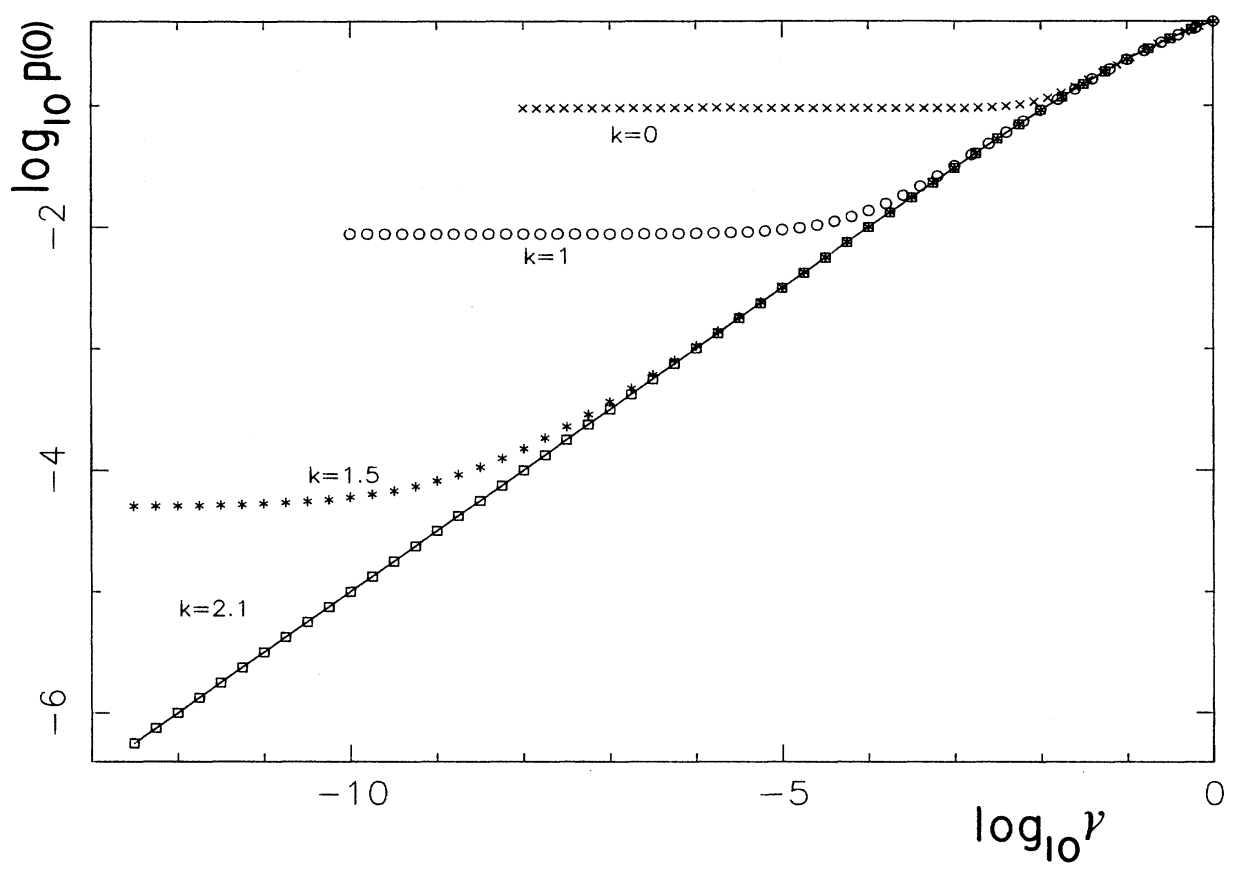

FIG. 1. Evolution of the upper level population as a function of the transition probability for the power cooling law, Eq. (4.1), for several values of the exponent $k$. All the curves correspond to $r=10^{-4}$ and $\mu=0.5$. The continuous line is the equilibrium population for each value of $\gamma$.

$$
\begin{gathered}
p(0)=\left[p_{0}-\frac{\gamma_{0}^{\mu}}{1+\gamma_{0}^{\mu}}\right] \exp \left[-\frac{1}{r}\left[\frac{\gamma_{0}^{2-k}}{2-k}+\frac{\gamma_{0}^{2+\mu-k}}{2+\mu-k}\right]\right] \\
+\mu \int_{0}^{\gamma_{0}} d y \frac{y^{\mu-1}}{\left(1+y^{\mu}\right)^{2}} \\
\quad \times \exp \left[-\frac{1}{r}\left[\frac{y^{2-k}}{2-k}+\frac{y^{2+\mu-k}}{2+\mu-k}\right]\right]
\end{gathered}
$$

This expression will be analyzed in the slow cooling limit, that according to Eq. (3.3) is characterized by

$$
\delta^{-1}=\frac{\gamma_{0}^{2-k}}{(2-k) r} \gg 1
$$

that implies $\left(\gamma_{0} \leq 1\right)$

$$
\rho=(2-k) r \ll 1
$$

The first term on the right-hand side of Eq. (4.7) is of order $e^{-1 / \delta}$. From now on, terms of this order will be neglected, since the behavior we are going to obtain is dominant against them. By doing the change of variable

$$
u=\frac{y^{2-k}}{(2-k) r}
$$

one gets

$$
\begin{aligned}
p(0) \sim v \rho^{v} \int_{0}^{1 / \delta} & d u \frac{u^{v-1}}{\left(1+\rho^{v} u^{v}\right)^{2}} \\
& \times \exp \left[-u\left[1+\frac{1}{1+v} \rho^{v} u^{v}\right]\right],
\end{aligned}
$$

where we have introduced

$$
v=\frac{\mu}{(2-k)} \text {. }
$$

The integral in Eq. (4.11) can be extended up to infinity with an exponentially small error. Next, we use the series expansions

$$
\exp \left[-\frac{\rho^{v}}{1+v} u^{1+v}\right]=\sum_{m=0}^{\infty} \frac{(-1)^{m}}{m !} \frac{\rho^{v m}}{(1+v)^{m}} u^{(1+v) m}
$$

and

$$
\left(1+\rho^{v} u^{v}\right)^{-2}=\sum_{n=0}^{\infty}(-1)^{n}(n+1) \rho^{v n} u^{v n} .
$$

The first one has an infinite radius of convergence, and the second one converges for $\rho u<1$. The maximum value of $u$ in Eq. (4.1) is $\delta^{-1}$ and, using Eq. (4.8) we get that $\rho u$ is bounded by $\gamma_{0}^{2-k}$, that is smaller than one for $k<2$. After introducing Eqs. (4.13) and (4.14) in Eq. (4.11) it is obtained

$$
\begin{aligned}
p(0) \sim v \rho^{v} \sum_{n=0}^{\infty} \sum_{m=0}^{\infty} & (-1)^{n+m} \frac{n+1}{m !(1+v)^{m}} \\
& \times \rho^{v(n+m)} \Gamma[v(1+n+m)+m] .
\end{aligned}
$$


The series is clearly convergent for $\rho<1$. In order to take the slow cooling limit, Eq. (4.9), two different cases must be considered. If $v$ is not too small, so that $\rho^{v} \ll 1$, we can keep only the first term in Eq. (4.15),

$$
p(0) \sim \rho^{v} \Gamma(1+v) .
$$

Nevertheless, if $v$ is so small that $\rho^{v}$ is of order of unity, we have to take a distinguishable limit. We rewrite Eq. (4.15) as

$$
\begin{array}{r}
p(0) \sim v \rho^{v} \sum_{n=0}^{\infty}(-1)^{n}(n+1) \rho^{v n} \Gamma[v(1+n)] \\
+v \rho^{v} \sum_{n=0}^{\infty} \sum_{m=1}^{\infty}(-1)^{n+m} \frac{n+1}{m !(1+v)^{m}} \\
\times \rho^{v(n+m)} \Gamma[v(1+n+m)+m] .
\end{array}
$$

Using the properties of the $\Gamma$ function it is seen that in the limit $v \rightarrow 0$ the second term on the right-hand side of Eq. (4.17) behaves as

$$
-\frac{v \rho^{v}}{\left(1+\rho^{v}\right)^{2}} \ln \left(1+\rho^{v}\right)
$$

and it goes to zero. For the first term one gets

$$
\rho^{v} \sum_{n=0}^{\infty}(-1)^{n} \rho^{v n}=\frac{\rho^{v}}{1+\rho^{v}}
$$

that is dominant as compared with Eq. (4.18). Therefore, for $v \rightarrow 0$,

$$
p(0) \sim \frac{\rho^{v}}{1+\rho^{v}}
$$

Matching the solutions given by Eqs. (4.16) and (4.20) it is found, uniformly in $v$,

$$
p(0) \sim p^{v} \Gamma(1+v)-\frac{\rho^{2 v}}{1+\rho^{v}},
$$

or

$$
\begin{aligned}
p(0) \sim & {[(2-k) r]^{\mu /(2-k)} \Gamma\left[1+\frac{\mu}{2-k}\right) } \\
& -\frac{[(2-k) r]^{2 \mu /(2-k)}}{1+[(2-k) r]^{\mu /(2-k)}} .
\end{aligned}
$$

This expression is uniformly valid in $\mu$. In particular, for $v=0$ it reduces to $p(0)=1 / 2$, showing that if there is no asymmetry the TLS always tends towards equilibrium, with the same population in both states. Of course, we cannot really speak of the RP in this case. In Fig. 2 the asymptotic behavior given by Eq. (4.22) is compared with the numerical evaluation of the exact expression, Eq. (4.7), for $k=1$ and $\mu=4$. It is seen that the agreement is surprisingly good over a wide range of values of the cooling rate $r$. In fact, on the scale of the figure, significant discrepancies only appear for $r \simeq 0.1$. We have checked that the range of validity of the asymptotic expression is larger, the smaller the value of $\mu$ is.

Equation (4.22) deserves some comments. It clearly

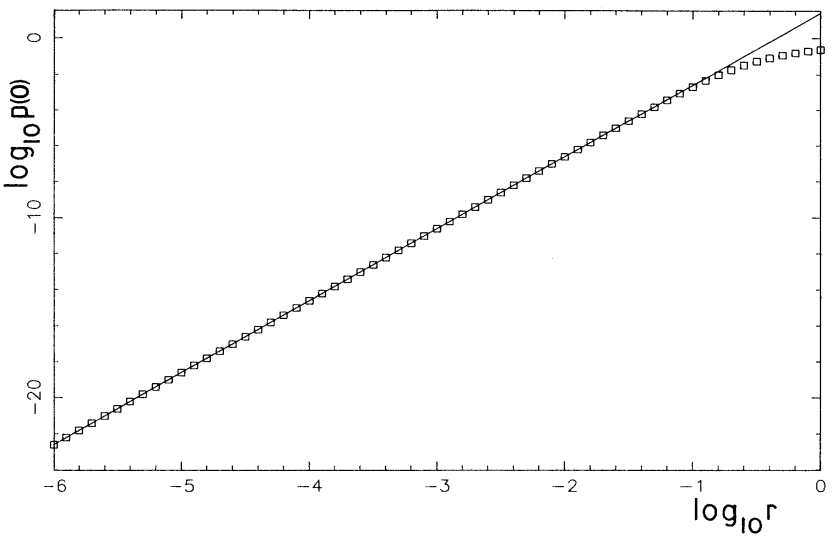

FIG. 2. Comparison of the exact (squares) and the asymptotic expressions for the RP for the cooling law $f(\gamma)=\gamma$. The value of the asymmetry parameter $\mu=4$.

shows that the RP depends on the cooling law, even in the slow cooling limit we are considering. In fact, we have proved in the previous section that the own existence of RP is determined by the way in which the temperature goes to zero, i.e., the value of $k$, and it is independent of the parameter $r$. The relevance of the value of $k$ shown in Fig. 3, where the RP is plotted as a function of $k$ for fixed $r$ and $\mu$. We have used both, the asymptotic expression, and also the exact one. The RP tends to zero as $k$ approaches the limit value $k=2$.

For $k<1$, the system is cooled to $T=0 \mathrm{~K}$ in a finite time $\tau$ given by Eq. (4.4), and the asymptotic RP depends on it, for a given $T_{0}$, in the form [for $\mu /(2-k)$ not too small]

$$
p(0) \propto \tau^{-\mu /(2-k)},
$$

where, again, the influence of the cooling law shows up. It must be noticed that Eq. (4.23) holds for all finite $\tau$, as long as the slow cooling condition, Eq. (4.8), is verified. Therefore, we can not speak of an universal dependence of the RP on the cooling time, even for very large $\tau$.

When $1 \leq k<2$ the system needs an infinite time to reach $T=0 \mathrm{~K}$, but there is always RP. For $\delta<<1$ $(\rho<<1)$ the RP is given by Eq. (4.22). When $k$ approaches the value 2 from below, the slow cooling condition does not require $r$ to be very small. In fact, for arbitrarily large $r$, there is always a range of values of $k$ in which the RP is asymptotically given by Eq. (4.22). Finally, for $k \geq 2$ there is no RP. More explicitly, for $k \geq 2$ the system is always at equilibrium, as seen in Sec. III.

\section{LINEAR COOLING}

A linear decrease of the temperature corresponds to a function $f(\gamma)$ given by Eq. (2.8) and, therefore, it cannot be reduced to the power laws discussed in the previous section. We already know that in this case there is $\mathbf{R P}$ and, from Eq. (2.16) we have 


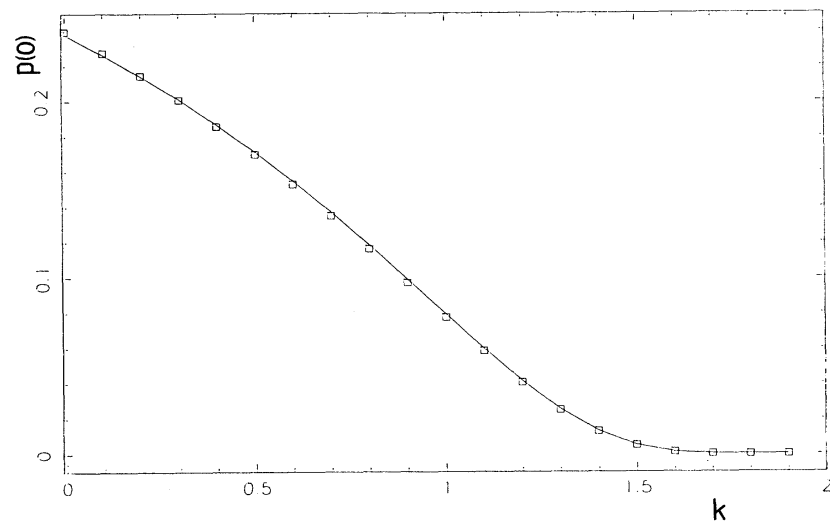

FIG. 3. Dependence of the RP on the value of the exponent $k$ for a power cooling law. The squares are the exact values and the continuous line corresponds to the asymptotic expression, Eq. (4.22). The cooling rate and the asymmetry parameter are $r=0.01$ and $\mu=0.5$, respectively.

$$
\begin{aligned}
p(0)= & p_{0} \exp \left[-\frac{1}{r} \int_{0}^{\gamma_{0}} d y \frac{1+y^{\mu}}{(\ln y)^{2}}\right] \\
& +\frac{1}{r} \int_{0}^{\gamma_{0}} d y \frac{y^{\mu}}{(\ln y)^{2}} \exp \left[-\frac{1}{r} \int_{0}^{y} d y^{\prime} \frac{1+y^{\prime \mu}}{\left(\ln y^{\prime}\right)^{2}}\right] .
\end{aligned}
$$

The slow cooling general condition expressed by Eq. (3.3) is equivalent, for not very high initial temperatures, to

$$
r<1 \text {. }
$$

This will be the asymptotic limit we will consider in this section. Thus, the initial term in Eq. (5.1) is exponentially small, and we can write

$$
p(0)=\frac{1}{r} \int_{0}^{\gamma_{0}} d y \frac{y^{\mu}}{(\ln y)^{2}} \exp \left[-\frac{1}{r} \int_{0}^{y} d y^{\prime} \frac{1+y^{\prime \mu}}{\left(\ln y^{\prime}\right)^{2}}\right] .
$$

The asymptotic analysis of this expression requires some care, since the integrand presents a rather broad moving maximum. ${ }^{7}$ We start by substituting $y=z r$ and thus obtain

$$
\begin{array}{rl}
p(0)=r^{\mu} \int_{0}^{\gamma_{0} / r} & d z \frac{z^{\mu}}{(\ln z+\ln r)^{2}} \\
& \times \exp \left[-\int_{0}^{z} d z^{\prime} \frac{1+r^{\mu} z^{\prime \mu}}{\left(\ln z^{\prime}+\ln r\right)^{2}}\right] .
\end{array}
$$

This expression is approximated by

$$
\begin{aligned}
p(0) \sim \frac{r^{\mu}}{(\ln r)^{2}} \int_{|\ln r|^{-1}}^{|\ln r|^{4}} d z z^{\mu} & {\left[1+\frac{\ln z}{\ln r}\right)^{-2} } \\
\times \exp & \left(-\int_{0}^{z} d z^{\prime} \frac{1+r^{\mu} z^{\prime} \mu}{\left(\ln z^{\prime}+\ln r\right)^{2}}\right),
\end{aligned}
$$

with an error that can be shown to be bounded by

$$
\left|I_{1}(r)\right|+\left|I_{z}(r)\right|
$$

where

$$
I_{1}(r)=\frac{r^{\mu}}{|\ln r|^{\mu+3}}
$$

and

$I_{2}(r)=r^{\mu}\left(\frac{\gamma_{0}}{r}\right)\left[\frac{\gamma_{0}}{r}-|\ln r|^{4}\right) \frac{1}{\left(\ln \gamma_{0}\right)^{2}} \exp \left[-\frac{1}{2}(\ln r)^{2}\right]$.

Let us study now the exponential appearing in the integrand of Eq. (5.5), i.e.,

$$
A(r)=\int_{0}^{z} d z^{\prime} \frac{1+r^{\mu} z^{\prime \mu}}{\left(\ln z^{\prime}+\ln r\right)^{2}} .
$$

The contribution to $A(r)$ coming from the region $0 \leq z^{\prime} \leq|\ln r|^{-2}$ is bounded by

$$
A_{1}(r)=\frac{1}{(\ln r)^{4}}
$$

and we will see it is negligible as compareed to the contribution from $|\ln r|^{-2} \leq z^{\prime} \leq z$. Thus,

$$
\begin{aligned}
A(r) & \sim \frac{1}{(\ln r)^{2}} \int_{|\ln r|^{-2}}^{z} d z^{\prime}\left[1+\frac{\ln z^{\prime}}{\ln r}\right]^{-2}\left(1+r^{\mu} z^{\prime \mu}\right) \\
& =\frac{1}{(\ln r)^{2}} \sum_{n=0}^{\infty}(-1)^{n} \int_{|\ln r|^{-2}}^{z} d z^{\prime}\left(1+r^{\mu} z^{\prime \mu}\right)(n+1)\left(\frac{\ln z^{\prime}}{\ln r}\right)^{n} .
\end{aligned}
$$

Taking into account that $|\ln r|^{-1} \leq z \leq|\ln r|^{4}$ it is easily verified that only the term $n=0$ must be kept and, after carrying out the integral, we obtain

$$
A(r) \sim \frac{1}{(\ln r)^{2}}\left[z+\frac{r^{\mu} z^{1+\mu}}{1+\mu}\right] .
$$

Now, it can be checked that $A_{1}$ is really negligible. In this way, we have arrived to

$$
\begin{aligned}
p(0) \sim \frac{r^{\mu}}{(\ln r)^{2}} \int_{|\ln r|^{-1}}^{|\ln r|^{4}} d z z^{\mu} & {\left[1+\frac{\ln z}{\ln r}\right]^{-2} } \\
\times \exp & {\left[-\frac{1}{(\ln r)^{2}}\left[z+\frac{r^{\mu} z^{1+\mu}}{1+\mu}\right]\right] . }
\end{aligned}
$$

Using again the same Taylor expansion as in Eq. (5.11) 
it is clear that

$$
\begin{aligned}
p(0) \sim & \frac{r^{\mu}}{(\ln r)^{2}} \\
& \times \int_{|\ln r|^{-1}}^{|\ln r|^{4}} d z z^{\mu} \\
& \quad \times \exp \left[-\frac{1}{(\ln r)^{2}}\left[z+\frac{r^{\mu} z^{1+\mu}}{1+\mu}\right]\right) .
\end{aligned}
$$

The range of the integral in Eq. (5.14) can be extended to $0 \leq z \leq \infty$ with an error smaller than

$$
\left|I_{3}(r)\right|+\left|I_{4}(r)\right| \text {, }
$$

where

$$
I_{s}(r)=\frac{r^{\mu}}{|\ln r|^{3+\mu}},
$$

and

$$
I_{4}(r)=r^{\mu}\left(1+\mu|\ln r|^{-2}+\cdots\right) \exp \left(-|\ln r|^{2}\right) .
$$

Thus, substitution of $s=z|\ln r|^{-2}$ yields

$$
\begin{aligned}
p(0) \sim & r^{\mu}|\ln r|^{2 \mu} \\
& \times \int_{0}^{\infty} d s s^{\mu} \exp \left[s\left[1+\frac{1}{1+\mu}|\ln r|^{2 \mu} r^{\mu} s^{\mu}\right]\right] .
\end{aligned}
$$

To evaluate the integral uniformly in $\mu$ we consider two regions. For $r^{\mu}|\ln r|^{2 \mu} \ll<1$ we get

$$
p(0) \sim r^{\mu}|\ln r|^{2 \mu} \Gamma(1+\mu)
$$

while for $r^{\mu}|\ln r|^{2 \mu}=0(1)$, that implies $\mu \rightarrow 0$, it is

$$
p(0) \sim \frac{r^{\mu}|\ln r|^{2 \mu}}{1+r^{\mu}|\ln r|^{2 \mu}} .
$$

Matching the two solutions, Eqs. (5.19) and (5.20), we obtain the final result, that is uniformly valid in $\mu$,

$$
p(0) \sim r^{\mu}|\ln r|^{2 \mu} \Gamma(1+\mu)-\frac{r^{2 \mu}|\ln r|^{4 \mu}}{1+r^{\mu}|\ln r|^{2 \mu}} .
$$

Besides, the terms $I_{1}, I_{2}, I_{3}$, and $I_{4}$, given by Eqs. (5.7), (5.8), (5.16), and (5.17), respectively, are clearly subdominant with respect to the contributions retained in Eq. (5.21).

Different asymptotic expressions for the RP after linear cooling have been previously derived by Huse and Fisher ${ }^{2}$ and by Langer, Dorsey, and Sethna. ${ }^{4}$ The one obtained by the latter authors reduces to Eq. (5.21) after neglecting terms that are of the same order as others that have already been omitted. The discrepancies with the result by Huse and Fisher are deeper.

The cooling time is given by

$$
\tau=-\frac{1}{r \ln \gamma_{0}}=\frac{k_{B} T_{0}}{V r},
$$

and for $\mu$ not too small

$$
p(0) \alpha \tau^{-\mu}|\ln \tau|^{2 \mu} .
$$

Therefore, the dependence of the RP on the cooling time is quite different from that found in the previous section (for $k<1$ ). We have again a proof of the relevance of the cooling procedure to determine the residual properties.

\section{RESIDUAL ENTROPY}

Two different, and nonequivalent, definitions of entropy will be considered here. First, there is the usual definition of statistical entropy, that for the TLS reads

$$
S=-k_{B}[p \ln p+(1-p) \ln (1-p)]
$$

and, second, we can define a calorimetric entropy by

$$
S_{2}^{\mathrm{cal}}-S_{1}^{\mathrm{cal}}=\int_{1}^{2} \frac{d Q}{T}
$$

where $d Q$ is the heat flow into the system. In our case, Eq. (6.2) reduces to ${ }^{6}$

$$
\frac{d S^{\mathrm{cal}}}{d t}=\frac{\varepsilon}{T(t)} \frac{d p}{d t} .
$$

It has been shown ${ }^{5,6}$ that when the above definitions are used to compute the residual entropy at $T=0 \mathrm{~K}$, starting from a state whose entropy is known, the residual calorimetric entropy (RCE) provides a lower bound for the residual statistical entropy (RSE). The aim of this section is to compute both, the RCE and the RSE, for a two-level system in the asymptotic limit of slow cooling. It will be assumed that the cooling law is expressed by a power of $\gamma$, i.e., it corresponds to Eq. (4.1). Besides, we will consider $k<2$, since we know that for $k \geq 2$ there is no RP, and, therefore, the RSE vanishes.

The calculation of the RSE is straightforward from its own definition and from the results in Sec. IV. Substitution of Eq. (4.21) into Eq. (6.1) yields

$$
\begin{aligned}
S(0) \sim & -k_{B}\left[\rho^{v} \Gamma(1+v)-\frac{\rho^{2 v}}{1+\rho^{v}}\right] \\
\times & \ln \left[\rho^{v} \Gamma(1+v)-\frac{\rho^{2 v}}{1+\rho^{v}}\right] \\
- & k_{B}\left[1-\rho^{v} \Gamma(1+v)+\frac{\rho^{2 v}}{1+\rho^{v}}\right] \\
& \times \ln \left[1-\rho^{v} \Gamma(1+v)+\frac{\rho^{2 v}}{1+\rho^{v}}\right),
\end{aligned}
$$

where the argument of $S$ refers to $\gamma=0$. For $\rho \ll<1$ and $v$ not too small Eq. (6.4) is equivalent to

$S(0) \sim-k_{B} \rho^{v} \Gamma(1+v)\left[\ln \rho^{v}+\ln \Gamma(1+v)-1\right]$.

On the other hand, for $\rho \ll 1$ and $\left.v \ll<1 \rho^{v}=0(1)\right]$ one gets

$S(0) \sim-k_{B} \frac{\rho^{v}}{1+\rho^{v}}\left[\ln \rho^{v}-\ln \left(1+\rho^{v}\right)-\rho^{-v} \ln \left(1+\rho^{v}\right)\right]$.

Matching Eqs. (6.5) and (6.6) in the overlapping region the following asymptotic expression, uniformly valid in $v$, 
is found for the RSE:

$$
\begin{aligned}
\frac{S(0)}{k_{B}} \sim & -p(0) \ln \rho^{v}+\left[\ln \left(1+\rho^{v}\right)-\rho^{v}\right] \\
& -\rho^{v} \Gamma(1+v)[\ln \Gamma(1+v)-1],
\end{aligned}
$$

with $p(0)$ given by Eq. (4.21).

Next we proceed to the calculation of the calorimetric entropy. Eq. (5.3) can be written in terms of the transition probability $\gamma$ as

$$
\frac{d S^{\mathrm{cal}}}{d \gamma}=-k_{B} \mu \ln \gamma \frac{d p}{d \gamma}
$$

To compute $S^{\text {cal }}$ from this equation we must specify an initial condition. We assume that at $t=0$ the system was at equilibrium with a transition probability $\gamma_{0}$. Thus, substitution of Eq. (4.1) into Eq. (2.17) and subsequent derivation leads to

$$
\begin{aligned}
\frac{d p}{d \gamma}= & \frac{\mu}{r} \gamma^{1-k}\left(1+\gamma^{\mu}\right) \exp \left[\frac{1}{r}\left[\frac{\gamma^{2-k}}{2-k}+\frac{\gamma^{2+\mu-k}}{2+\mu-k}\right]\right] \\
& \times \int_{\gamma}^{\gamma_{0}} d y \frac{y^{\mu-1}}{\left(1+y^{\mu}\right)^{2}} \\
& \times \exp \left[-\frac{1}{r}\left[\frac{y^{2-k}}{2-k}+\frac{y^{2+\mu-k}}{2+\mu-k}\right]\right] .
\end{aligned}
$$

This expression is introduced into Eq. (6.8), that is then integrated between $\gamma_{0}$ and 0 . In the Appendix it is shown that after some algebra one obtains for the residual entropy

$$
\begin{aligned}
S^{\mathrm{cal}}(0)= & -k_{B} v p(0) \ln \rho-k_{B} v^{2} \rho^{v} \int_{0}^{1 / \delta} d z \frac{z^{v-1}}{\left(1+\rho^{v} z^{v}\right)^{2}} \exp \left[-z\left[1+\frac{\rho^{v} z^{v}}{1+v}\right)\right] \ln z \\
& +k_{B} v^{2} \rho^{v} \int_{0}^{1 / \delta} d z \frac{z^{v}}{\left(1+\rho^{v} z^{v}\right)^{2}} \int_{0}^{1} d u \ln (1-u)\left[1+\rho^{v} z^{v}(1-u)^{v}\right] \\
& \times \exp (-z u) \exp \left[-z \frac{\rho^{v} z^{v}}{1+v}\right]\left[z(1-u) \frac{\rho^{v} z^{v}(1-u)^{v}}{1+v}\right],
\end{aligned}
$$

with $\delta, \rho$, and $v$ given by Eqs. (4.8), (4.9), and (4.12), respectively. Eq. (6.10) is exact, and holds for arbitrary values of all the parameters, with the only restriction $k<2$. Let us study its asymptotic behavior in the slow cooling limit. For $\rho<<1$ and $v$ not too small one finds

$$
S^{\mathrm{cal}}(0) \sim-k_{B} \rho^{v} \Gamma(1+v)\left[\ln \rho^{v}+v \psi(v)\right]+k_{B} v^{2} \rho^{v} \int_{0}^{\infty} d z z^{v} \int_{0}^{1} d u \ln (1-u) e^{-z u}
$$

where $\psi$ is the digamma function. ${ }^{8}$ For $\rho \ll 1$ and $v<<1\left[\rho^{v}=0(1)\right]$, a naive approximation of the second term in the right-hand side (rhs) of Eq. (6.10) leads to the divergent integral

$$
\int_{0}^{\infty} d z \frac{z^{-1}}{\left(1+\rho^{v}\right)^{2}} \exp \left[-z\left(1+\rho^{v}\right)\right] \ln z
$$

In order to carry out a more careful analysis we expand $\left(1+\rho^{v} z^{v}\right)^{-2}$ and $\exp \left[-\rho^{v} z^{1+v} /(1+v)\right]$. Also, it is easily realized that the integrals can be extended up to infinity with a negligible error. In this way, after some algebra one obtains

$$
\begin{aligned}
v^{2} \int_{0}^{1 / \delta} d z \frac{z^{v-1}}{\left(1+\rho^{v} z^{v}\right)^{2}} \exp \left[-z\left[1+\frac{\rho^{v} z^{v}}{1+v}\right]\right] \ln z & \\
& \sim \sum_{m=0}^{\infty}(-1)^{m+1} \frac{\rho^{v m}}{m+1} \Gamma(v+v m+1)+v \sum_{m=0}^{\infty}(-1)^{m} \rho^{v m} \Gamma(v+v m+1) \psi(v+v m+1) \\
& +v^{2} \sum_{m=0}^{\infty} \sum_{n=1}^{\infty} \frac{(-1)^{m+n}(m+1) \rho^{v(m+n)}}{n !(1+v)^{n}} \Gamma[v(1+m+n)+n] \psi[v(1+m+n)+n]
\end{aligned}
$$

For $v \rightarrow 0$, the dominant contribution is given by the first summation that reduces to 


$$
\rho^{-v} \sum_{s=1}^{\infty} \frac{(-1)^{s}}{s} \rho^{v s}=-\rho^{-v} \ln \left(1+\rho^{v}\right)
$$

The third and fourth terms on the right-hand side of Eq. (6.10) are asymptotically much smaller than this. Thus, the asymptotic behavior of Eq. (6.10) in the limit $\rho \rightarrow 0$ and $v \rightarrow 0$ is described by

$$
S^{\mathrm{cal}}(0) \sim-k_{B} \frac{\rho^{v}}{1+\rho^{v}} \ln \rho^{v}+k_{B} \ln \left(1+\rho^{v}\right) .
$$

To have an expression valid uniformly in $v$, we look for a common limit for Eqs. (6.11) and (6.15) in an overlapping region, for instance,

$$
|\ln r|^{-1 / 2}<v<|\ln r|^{-1 / 3} .
$$

The common limit is

$$
S(0) \sim-k_{B} \rho^{v} \ln \rho^{v}+k_{B} \rho^{v},
$$

and, therefore, our final result for the RCE (uniformly in v) reads

$$
\begin{aligned}
\frac{S^{\mathrm{cal}}(0)}{k_{B}} \sim & -p(0) \ln \rho^{v}+\ln \left(1+\rho^{v}\right) \\
& -\rho^{v}+\rho^{v} \Gamma(1+v)[v \psi(1+v)-1] \\
& +v^{2} \rho^{v} \int_{0}^{\infty} d z z^{v} \int_{0}^{1} d u \ln (1-u) e^{-z u}
\end{aligned}
$$

We see that both, the RSE and RCE, depend on the cooling law. Nevertheless, for cooling laws of the form $\dot{\gamma}(t)=-r \gamma^{k}$ they can be expressed in a form that is independent from $k$ by introducing an effective cooling rate

$$
\rho=(2-k) r
$$

and an effective asymmetry parameter

$$
v=\frac{\mu}{2-k} \text {. }
$$

A similar result was found for the RP in Sec. IV.

Given that the RSE and the RCE have been computed in the same order of approximation, it is meaningful to consider their difference,

$$
\begin{aligned}
S(0)- & S^{\mathrm{cal}}(0) \\
\sim & k_{B} \rho^{v} \Gamma(1+v)[v \psi(1+v)-\ln \Gamma(1+v)] \\
& \quad-k_{B} v^{2} \rho^{v} \int_{0}^{\infty} d z z^{v} \int_{0}^{1} d u \ln (1-u) e^{-z u}
\end{aligned}
$$

The last term in this expression is defined positive due to the range of integration of $u$. Besides, ${ }^{8}$

$v \psi(1+v)-\ln \Gamma(1+v)=\int_{0}^{v} d x x \psi^{(1)}(x+1)>0$,

where $\psi^{(1)}(x)$ is the trigamma function

$$
\psi^{(1)}(x)=\int_{0}^{\infty} d t \frac{t e^{-x t}}{1-e^{-t}},
$$

that is defined positive for $x \geq 1$. In this way, we have proved that

$$
S(0)-S^{\mathrm{cal}}(0)>0,
$$

uniformly in $v$. The result is consistent with the more general one derived in Refs. 5 and 6.

The difference between the RSE and the RCE increases with the value of the effective asymmetry parameter $v$, and it vanishes when $v$ goes to zero. For $v=0$ it follows from Eqs. (6.7) and (6.18) that $S(0)=S^{\mathrm{cal}}(0)=k_{B} \ln 2$. We use the equality sign here, since it can be easily proved that this is an exact result. In fact, the statistical and calorimetric entropies remain constant along the cooling process of the TLS in this case.

A comparison of Eqs. (4.22) and (6.21) shows that the difference of entropies is of the same order in the effective cooling rate $\rho$ as the residual population or the residual energy. If this were also true in real glasses, one could expect significant differences between the measured RCE and the theoretical RSE obtained from a definition in terms of the probability density.

\section{CONCLUSIONS}

A necessary and sufficient condition for the existence of a residual population when the temperature of an ensemble of identical two-level systems is lowered to $T=0$ $\mathrm{K}$ has been obtained. The condition is given by Eq. (3.12) or, in terms of the temperature, by

$$
\lim _{T \rightarrow 0} \frac{1}{T}\left|\frac{\partial \ln T}{\partial t}\right| \exp \left[\frac{V}{k_{B} T}\right]=\infty .
$$

It is, therefore, the behavior of the cooling law near $T=0$ that determines the possibility of a residual population.

Also, a precise definition of slow cooling has been introduced. It involves the cooling law, the cooling rate parameter and the initial condition. In the asymptotic limit of slow cooling, we have derived explicit expressions for the residual population and entropy. The results show a clear dependence on the cooling law, contrary to what has been stated by some authors.

In particular, the simple picture that the population freezes when the rate of cooling equals the transition rate does not lead to the correct qualitative behavior. Instead, the picture that emerges form our analysis is that the freezing takes place when the average number of transitions that the TLS is going to experiment until reaching $T=0 \mathrm{~K}$ is of the order of unity. Also, the system will have forgotten its initial conditions after a time allowing a large enough number of transitions. That means that the relevant time scale is given by

$$
d s=-\gamma(t) d t \text {. }
$$

as suggested by Eq. (2.4). The origin of $s$ is taken such that $s=0$ for $\gamma=0$, i.e.,

$$
s=\int_{t}^{t_{0}} d t^{\prime} \gamma\left(t^{\prime}\right)
$$

where $t_{0}$ is the time for which $\gamma$ (and $T$ ) vanishes. The value of $s$ is proportional to the average number of transitions from $t$ to $t_{0}$.

If $s(0) \gg 1$ the system has time to reach equilibrium before getting frozen. This can be understood as the con- 
dition for slow cooling. It follows that the residual population will be roughly of the order of the equilibrium population corresponding to $s=1$. For the power cooling law given by Eq. (4.1) one gets

$$
\gamma(s=1)=[(2-k) r]^{1 /(2-k)} .
$$

and the slow cooling condition reads $(2-k) r \ll 1$, that agrees with Eq. (4.9). It can be checked that Eq. (7.4) correctly predicts the values of $\gamma$ for which the separation of equilibrium and the freezing take place in Fig. 1. From Eq. (7.4) it follows,

$$
p(0) \sim \frac{[(2-k) r]^{\mu /(2-k)}}{1+[(2-k) r]^{\mu /(2-k)}} .
$$

For $\mu /(2-k)<<1$, Eq. (7.5) reduces to the exact asymptotic behavior, Eq. (4.20), while for $\mu /(2-k)$ not too small one gets

$$
p(0) \sim[(2-k) r]^{\mu /(2-k)}
$$

that also agrees with the exact asymptotic behavior, except for a factor of the order of unity.

The linear cooling case can be discussed in a similar way. It is easily found that

$$
\gamma(s=1) \sim r(\ln r)^{2}
$$

and the slow cooling condition reads $\left.r(\ln r)^{2}<<1\right)$. From here one easily reobtains Eqs. (5.19) and (5.20), the former without the function $\Gamma$ factor.

Starting from the analysis presented here, it is straightfoward to study the asymptotic residual properties of an ensemble of two-level systems with a given distribution of excitation energies and activation barriers. The results will be presented in a follow-up paper.

\section{ACKNOWLEDGMENTS}

We acknowledge partial support from the Dirección General de Investigación Cientifica y Técnica (Spain) through Grant No. PB89-0618.

\section{APPENDIX}

In this appendix we derive Eq. (6.10), which gives an exact expression for the calorimetric entropy at $T=0 \mathrm{~K}$ when the TLS is cooled with a law given by Eq. (4.1), with $k<2$. From Eqs. (6.8) and (6.9) we get

$$
\frac{d S^{\mathrm{cal}}}{d \gamma}=-k_{B} \frac{\mu^{2}}{r} \gamma^{1-k}\left(1+\gamma^{\mu}\right) \exp \left[\frac{1}{r}\left[\frac{\gamma^{2-k}}{2-k}+\frac{\gamma^{2+\mu-k}}{2+\mu-k}\right]\right] \int_{\gamma}^{\gamma_{0}} d y \frac{y^{\mu-1}}{\left(1+y^{\mu}\right)^{2}} \exp \left[-\frac{1}{r}\left[\frac{y^{2-k}}{2-k}+\frac{y^{2+\mu-k}}{2+\mu-k}\right]\right] \text {. }
$$

If the initial state was of equilibrium, by integration of Eq. (A1) we obtain

$$
\begin{array}{rl}
S^{\mathrm{cal}}(0)=S_{\mathrm{eq}}\left(\gamma_{0}\right)+k_{B} \frac{\mu^{2}}{r} \int_{0}^{\gamma_{0}} & d \gamma \gamma^{1-k}\left(1+\gamma^{\mu}\right) \exp \left[\frac{1}{r}\left[\frac{\gamma^{2-k}}{2-k}+\frac{\gamma^{2+\mu-k}}{2+\mu-k}\right]\right] \\
& \times \int_{\gamma}^{\gamma_{0}} d y \frac{y^{\mu-1}}{\left(1+y^{\mu}\right)^{2}} \exp \left[-\frac{1}{r}\left[\frac{y^{2-k}}{2-k}+\frac{y^{2+\mu-k}}{2+\mu-k}\right]\right] .
\end{array}
$$

The argument of $S^{\text {cal }}$ refers to $\gamma=0$, as in Sec. VI.

Changing the order of integration in the second term, and using the identity

$$
\ln \gamma=\ln \gamma+\frac{1}{2-k} \ln [(2-k) r]-\frac{1}{2-k} \ln [(2-k) r],
$$

one gets

$$
\begin{aligned}
S^{\mathrm{cal}}(0)= & S_{\mathrm{eg}}\left(\gamma_{0}\right)+k_{B} \frac{\mu^{2}}{(2-k) r} \ln [(2-k) r] \int_{0}^{\gamma_{0}} d y \frac{y^{\mu-1}}{\left(1+y^{\mu}\right)^{2}} \exp \left[-\frac{1}{r}\left[\frac{y^{2-k}}{2-k}+\frac{y^{2+\mu-k}}{2+\mu-k}\right]\right] \\
& \quad \times \int_{0}^{y} d \gamma \gamma^{1-k}\left(1+\gamma^{\mu}\right) \exp \left[\frac{1}{r}\left[\frac{\gamma^{2-k}}{2-k}+\frac{\gamma^{2+\mu-k}}{2+\mu-k}\right]\right] \\
+ & k_{B} \frac{\mu^{2}}{(2-k) r} \int_{0}^{\gamma_{0}} d y \frac{y^{\mu-1}}{\left(1+y^{\mu}\right)^{2}} \exp \left[-\frac{1}{r}\left[\frac{y^{2-k}}{2-k}+\frac{y^{2+\mu-k}}{2+\mu-k}\right]\right] \\
& \times \int_{0}^{y} d \gamma \gamma^{1-k}\left(1+\gamma^{\mu}\right) \ln \left[\frac{\gamma^{2-k}}{(2-k) r}\right] \exp \left[\frac{1}{r}\left[\frac{\gamma^{2-k}}{2-k}+\frac{\gamma^{2+\mu-k}}{2+\mu-k}\right]\right] .
\end{aligned}
$$

The second integral in the second term on the RHS of Eq. (A4) can be easily evaluated, giving 


$$
\begin{aligned}
S^{\mathrm{cal}}(0)= & S_{\mathrm{eq}}\left(\gamma_{0}\right)-k_{B} \frac{\mu}{2-k} \ln [(2-k) r] p(0)+k_{B} \frac{\mu^{2}}{(2-k) r} \ln [(2-k) r] \int_{0}^{\gamma_{0}} d y \frac{y^{\mu-1}}{\left(1+y^{\mu}\right)^{2}} \\
& +k_{B} \frac{\mu^{2}}{(2-k) r} \int_{0}^{\gamma_{0}} d y \frac{y^{\mu-1}}{\left(1+y^{\mu}\right)^{2}} \exp \left[-\frac{1}{r}\left[\frac{y^{2-k}}{2-k}+\frac{y^{2+\mu-k}}{2+\mu-k}\right]\right] \\
& \times \int_{0}^{y} d \gamma \gamma^{1-k}\left(1+\gamma^{\mu}\right) \ln \left[\frac{\gamma^{2-k}}{(2-k) r}\right] \exp \left[\frac{1}{r}\left[\frac{\gamma^{2-k}}{2-k}+\frac{\gamma^{2+\mu-k}}{2+\mu-k}\right]\right] .
\end{aligned}
$$

Now, we introduce the new variables

$$
z=\frac{y^{2-k}}{(2-k) r}, \quad x=\frac{\gamma^{2-k}}{(2-k) r},
$$

and use the identity

$$
S_{\text {eq }}\left(\gamma_{0}\right)=-k_{B} \mu^{2} \int_{0}^{\gamma_{0}} d \gamma \ln \gamma \frac{\gamma^{\mu-1}}{\left(1+\gamma^{\mu}\right)^{2}}
$$

The result, in terms of the parameters $\rho, v, \delta$ defined in Sec. IV, is

$$
\begin{aligned}
S^{\mathrm{cal}}(0)= & -k_{B} v \ln \rho p(0)-k_{B} v^{2} \rho^{v} \int_{0}^{1 / \delta} d z \frac{z^{v-1}}{\left(1+\rho^{v} z^{v}\right)^{2}} \\
& \times\left\{\ln z-\exp \left[-z\left[1+\frac{1}{1+v} \rho^{v} z^{v}\right]\right] \int_{0}^{z} d x\left(1+\rho^{v} x^{v}\right) \ln x \exp \left[x\left[1+\frac{1}{1+v} \rho^{v} x^{v}\right]\right]\right\} .
\end{aligned}
$$

Now, we put $\ln x=\ln z+\ln (x / z)$, and rewrite the double integral in terms of $z$ and $t=x / z$. After some very simple algebraic manipulation, Eq. (A8) becomes

$$
\begin{aligned}
S^{\mathrm{cal}}(0)= & -k_{B} v p(0) \ln \rho-k_{B} v^{2} \rho^{v} \int_{0}^{1 / \delta} d z \frac{z^{v-1}}{\left(1+\rho^{v} z^{v}\right)^{2}} \exp \left[-z\left(1+\frac{\rho^{v} z^{v}}{1+v}\right)\right] \ln z \\
& +k_{B} v^{2} \rho^{v} \int_{0}^{1 / \delta} d z \frac{z^{v}}{\left(1+\rho^{v} z^{v}\right)^{2}} \exp \left[-z\left(1+\frac{\rho^{v} z^{v}}{1+v}\right)\right] \int_{0}^{1} d t \ln t\left(1+\rho^{v} z^{v} t^{v}\right) \exp \left[z t\left(1+\frac{\rho^{v} z^{v} t^{v}}{1+v}\right)\right] .
\end{aligned}
$$

To arrive to Eq. (6.10) we only have to introduce $u=1-t$.

${ }^{1}$ W. A. Phillips, J. Low. Temp. Phys. 7, 351 (1972).

${ }^{2}$ D. A. Huse and D. S. Fisher, Phys. Rev. Lett. 57, 2203 (1986).

${ }^{3}$ S. A. Langer and J. P. Sethna, Phys. Rev. Lett. 61, 570 (1988).

${ }^{4}$ S. A. Langer, A. T. Dorsey, and J. P. Sethna, Phys. Rev. B 40, 345 (1989).

${ }^{5}$ S. A. Langer, J. P. Sethna, and E. R. Grannan, Phys. Rev. B 41, 2261 (1990).
${ }^{6}$ J. J. Brey and A. Prados, Phys. Rev. A 41, 765 (1990).

${ }^{7}$ C. M. Bender and S. A. Orszag, Advanced Mathematical Methods for Scientists and Engineers (McGraw-Hill, New York, 1978).

${ }^{8}$ Handbook of Mathematical Functions, edited by M. A. Abramowitz and I. A. Stegun (Dover, New York, 1972). 